\title{
Sleep-Wake Disorders: Definition, Contexts and Neural Correlations
}

\section{Journal of \\ Neurology and Psychology}

\author{
Giulio Perrotta* \\ Department of Criminal and Investigative Psychology Studies, Italy \\ *Address for Correspondence \\ Giulio Perrotta, Department of Criminal and Investigative Psychology \\ Studies, University of Federiciana, Cosenza, Italy, Phone: (+39) 349 \\ 2108 872; E-mail: giuliosr1984@hotmail.it \\ Submission: May 23, 2019 \\ Accepted: July 05, 2019 \\ Published: July 08, 2019 \\ Copyright: (c) 2019 Perrotta G, et al. This is an open access article \\ distributed under the Creative Commons Attribution License, which \\ permits unrestricted use, distribution, and reproduction in any medium, \\ provided the original work is properly cited.
}

for the proper functioning of both" [5].

Yet another definition indicates it as: "A readily reversible state of reduced activity and interaction with the surrounding environment." Thus the term "readily reversible" cannot be associated with coma or anesthesia which, respectively, are pathology and a pharmacologically induced state of rest.

\section{Sleep therefore differs from other states of altered consciousness}

a) With sleep the abolition of the state of vigilance is, as already mentioned, reversible. Thus the subject can awaken after an even painless stimulus;

b) Otherwise, stupor is an alteration of the state of consciousness from which one can awaken only after administering a painful stimulus;

c) The comatose state is an alteration of the state of consciousness from which one cannot awaken after administering a painful stimulus;

d) Brain death is much more serious with the irreversible cessation of all brain activity.

Traditionally, three main measures have been used to define sleep physiology:

a) The electroencephalogram (conventionally abbreviated as "EEG") which translates brain activity into electrical waves;

b) The electrooculogram (conventionally abbreviated as "EOG") records eye movements and translates them into electric waves;

c) The electromyogram (conventionally abbreviated as "EMG") which records muscular activity (usually in polysomnography that of the mylohyoid muscle). For a thorough examination of sleep we use polysomnography, with which we record, during an entire night, a series of physiological parameters, such as the movement of the ribcage and the abdomen, the flow of air that passes through the oronasal cavity, blood oxygen saturation and heart rate.

In 1953, Eugene Aserinsky and Nathaniel Kleitman discovered the presence of Rapid Eye Movements (REM) during sleep. This simple observation made it possible to differentiate sleep in a REM phase (with rapid eye movements) and in a non-REM phase (NREM 


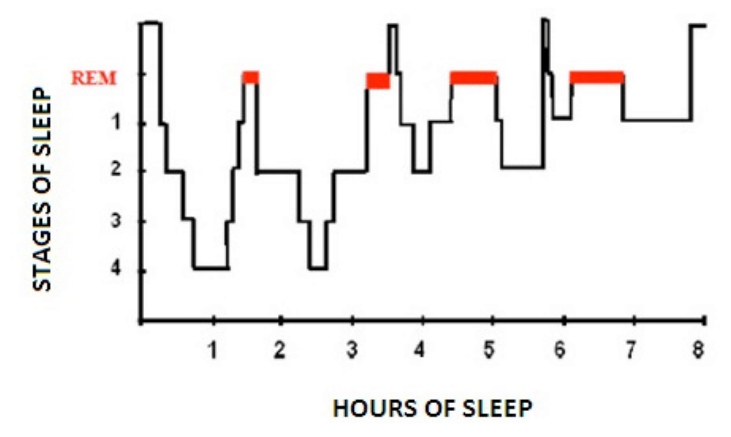

Figure 1: Hypnogram.

Source: https://www.researchgate.net/figure/Hypnogram-of-sleep-cycle-in-ahealthy-young-adult-Normal-sleep-involves-cycling_fig1_267910844

phase). In 1963, Kleitman and Dement described for the first time the alternation, during the period of sleep, of REM and NREM sleep in cycles, introducing the concept of sleep architecture. At the end of the 1960s, after the discovery of REM and NREM sleep and the concept of cyclical nature of these two phases within sleep, the need arose to classify the electroencephalographic changes that occurred during sleep in a macroscopic manner in a standard manner. In 1968, Rechtschaffen and Kales based on the analysis of electroencephalographic, electromyographic and electrooculographic parameters classified sleep in 5 stages: 4 NREM stages (stage 1; stage 2; stage 3; stage 4) and a REM stage (Figure 1).

Sleep presents a regular alternation of non-REM and REM phases consisting of cycles of similar duration to each other. After falling asleep the subject progressively passes from stage 1 of non-REM sleep to stage 2 , after which he passes to stage 3 or stage 4 and then, between 70 and 90 minutes after falling asleep, the first phase of REM sleep occurs which lasts about 15 minutes. At the end of the first phase of REM sleep the first cycle ends, which lasts approximately 80 to 100 minutes.

After the first cycle, others of a rather constant duration follow one another, but where REM sleep tends to increase in duration at the expense of non-REM sleep, in particular stages 3 and 4 (deep sleep) which become shorter. During the night, in the end, REM sleep constitutes about $25 \%$ of the total sleep duration. It is possible that there are waking moments between the various cycles. The period of sleep is represented graphically by the hypnograms that illustrate the succession of the phases of wakefulness and sleep in relation to time. Today in place of the subdivision into four stages the nomenclature in three phases (N1, N2 and N3) adopted by the American Academy of Sleep Medicine in 2007 on the basis of the appearance and frequencies of the EEG oscillations, in which phase N3 combines stages 3 and 4 both characterized by the same large slow waves, even if in different percentages.

During the vigil, the EEG basically alternates between two patterns. A pattern called "activation" (or desynchronized pattern) characterized by low voltage waves (10-30 micro volts) and high frequency $(16-25 \mathrm{~Hz})$ and a second called "alpha activity" characterized by sine waves of $8-12 \mathrm{~Hz}$ Alpha activity is typically present and abundant when the subject is relaxed with eyes closed. The activation pattern is present when the patient is in a state of attention with open eyes. Eye movements are both rapid and slow and muscle tone is medium to high.

During stage 1, the alpha activity decreases, the activation pattern is poor and the EEG consists mainly of low-voltage waves of mixed frequency between 3-7 Hz. The movements of the eyes are still present but slow, rotating and oscillators (not in phase opposition as in the REM phase). The electromyogram shows a persistent tonic activity although of a lower intensity than the wake. In stage 2 there is a relatively low background activity, with variable frequency but close to theta waves $(3-7 \mathrm{~Hz})$. Stage 2 is characterized by the presence of two components, the so-called $\mathrm{K}$ complexes and the spindles of sleep (or spindles). The latter of thalamic origin, lacking in lethal family insomnia, a deadly disease for sleep deprivation. The eye movements are slow, while the EMG is further reduced. In stage 3, 20\% - 50\% of each epoch (conventionally an EEG recording period of $30 \mathrm{~s}$ ) must contain Delta activities, i.e., large amplitude EEG waves ( $>75$ micro volts) and low frequency (about $0.5-4 \mathrm{~Hz}$ ). The muscle tone in this stage is slightly reduced and the eye movements practically absent. The spindles of sleep may or may not occur, while the K complexes are present, although they are often difficult to distinguish from delta waves. Stage 4 is characterized by the presence of delta waves, which here reach the maximum amplitude and minimum frequency, for more than $50 \%$ of each era. As for stage 3, the spindles can be absent or present while the K complexes are present, but almost unrecognizable from the underlying delta rhythm. The movements of the eyes are not present while a state of very low tonic muscle activation persists. At this stage the metabolic activity of the brain is reduced (lower consumption of oxygen and glucose). If the subject wakes up at this stage he can get confused for a few minutes (Figure 2-4).

The REM stage is characterized by a low voltage EEG with mixed frequencies. The EEG of REM sleep is very reminiscent of that of stage 1 if not for the discharged characteristics of waves with the characteristic 'saw-tooth' morphology. PGO (ponto-genicoccipital) waves appear, the activity of the hippocampus becomes synchronized with the appearance of theta waves. The stadium takes its name from rapid eye movements and the low tone of the mental

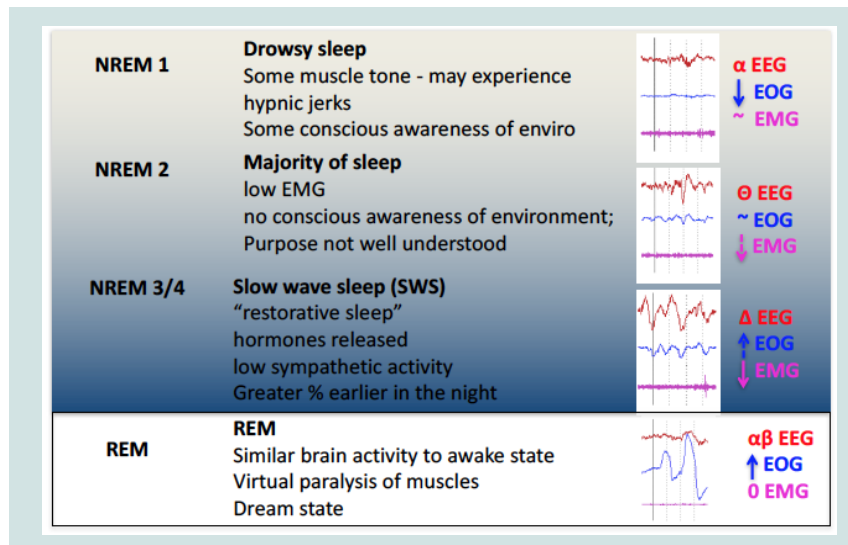

Figure 2: Sleepphisiology.

Part I. Source: https://foreverfitscience.com/sleep/sleep-physiology/ 


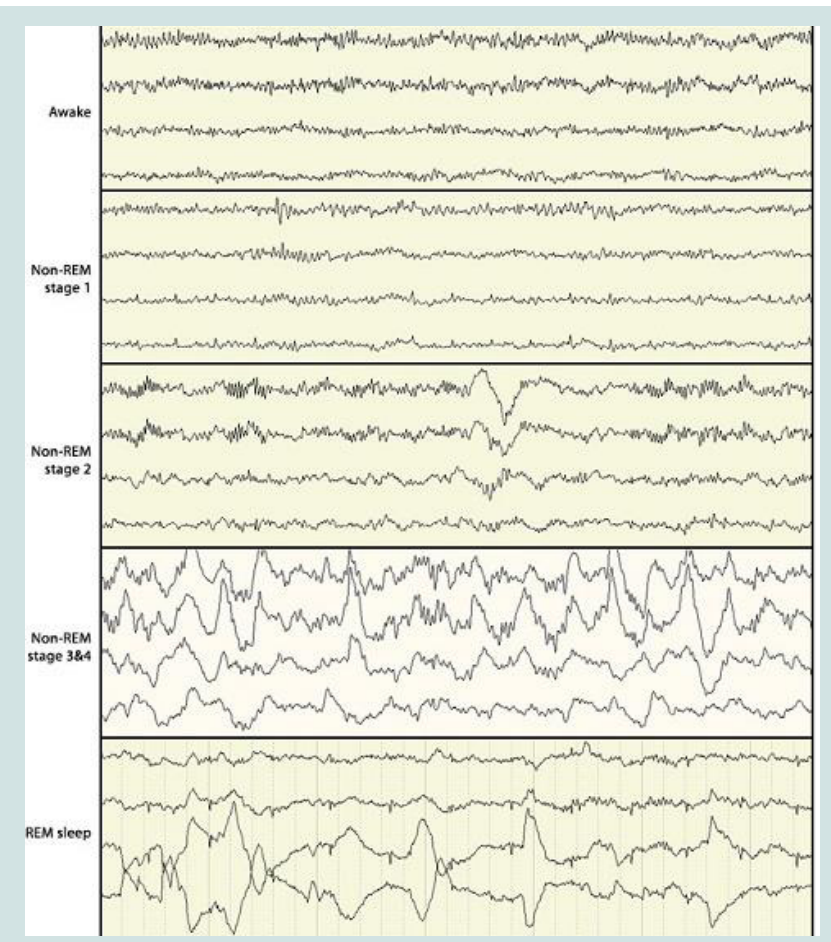

Figure 3: Sleepphisiology.

Part II. Source: https://healthengine.com.au/info/sleep-physiology

muscles. Moreover, this phase is characteristic for the paralysis of the muscles (to avoid mimicking dreams) and because it is the one in which dreams predominantly occur. The brain consumes oxygen and glucose as if the subject were awake and engaged in intellectual activity. If you wake up in this phase you are perfectly oriented. This stage is also characterized by a more imprecise control of the vegetative functions of the organism, in fact the arterial pressure increases and undergoes sudden changes, the heart rate increases and extra systoles can appear, the respiratory frequency becomes more irregular and the part is compromised thermo regulation. Penile erection in men and genital changes in women may occur. REM sleep tends to decrease with advancing age and reaches a peak at the age of 1 year and then decreases in favor of non-REM sleep.

Sleep deprivation was tested by Randy Gardner in 1965, a young 17 -year-old student who stayed awake for 264 hours, or 11 days. On the second day, his concentration diminished and subsequently lost the ability to identify objects by touch. At the end of the third day he experienced bad temper and disorientation. At the end of the experiment it was difficult to concentrate, to remember recent events; he became paranoid and began to hallucinate. On the eleventh day, the medical personnel who kept him under control wrote: "Confusional state and disorientation, sudden mood swings, irritability, speak with a road sign believing him a man, hallucinations, temporary loss of identity, difficulty in pronouncing tongue twisters, mumbles many words, diminished reflexes, memory lapses, difficulty in focusing objects, visual problems with too bright colors ... ". When we sleep little, in fact, learning, memory, mood and quick reflexes are compromised. Several studies show that sleeping systematically less than six hours a night increases the risk of heart attack for well over four and a half times compared to those who sleep regularly.

\section{Sleep-Wake Disorders: Classification and Clinical Contexts}

When we talk about these disorders we refer to a wide range of problems characterized by an alteration of the sleep-wake rhythm [6]. Those who present these disorders are unable to benefit from their rest and perceive their sleep as insufficient or unsatisfactory in quality and quantity, resulting in stress and discomfort during waking hours.

Due to the close connection between sleep quality and physical and emotional health, even when a sleep disorder occurs occasionally in a person, it should never be neglected. Sleep-related disorders are accompanied by clinically significant distress or impairment in the social, occupational or other important areas.

\section{Sleep-wake disorders include the following sub-categories with related symptoms}

Insomnia disorder: predominant dissatisfaction with the quantity or quality of sleep associated with difficulty in starting sleep at bedtime, maintaining sleep, with frequent or prolonged awakenings during the night and early morning awakening with difficulty go back to sleep. For there to be a diagnosis of insomnia disorder the difficulty of sleeping must occur at least 3 times a week and must persist for at least three months despite adequate sleeping conditions.

Hypersomnolence disorder: excessive sleepiness despite a main sleep period of at least 7 hours, manifesting at least three times a week for at least three months. It is accompanied by clinically significant distress or impairment in cognitive, social, occupational or other important areas. It is not attributable to the effects of a substance (a substance of abuse, a drug) and the complaint of sleep inessis not adequately explained by the coexistence of mental and clinical disorders. It is not justified by another sleep disorder and does not occur exclusively during the course of another sleep disorder.

Narcolepsy: It is characterized by recurrent periods of irrepressible bi-dream of sleeping, sleep attacks or naps that occur

\begin{tabular}{|c|c|c|}
\hline Physiological Process & NREM & REM \\
\hline Brain activity & Decreases from wakefulness & $\begin{array}{l}\text { Increases in motor and sensory } \\
\text { areas, while other areas are } \\
\text { similar to NREM }\end{array}$ \\
\hline Heart rate & Slows from wakefulness & $\begin{array}{l}\text { Increases and varies compared } \\
\text { to NREM }\end{array}$ \\
\hline Blood pressure & Decreases from wakefulness & $\begin{array}{l}\text { Increases (up to } 30 \text { percent) and } \\
\text { varies from NREMM }\end{array}$ \\
\hline Sympathetic nerve activity & Decreases from wakefulness & $\begin{array}{l}\text { Increases significantly from } \\
\text { wakefulness }\end{array}$ \\
\hline Muscle tone & Similar to wakefulness & Absent \\
\hline Blood flow to brain & Decreases from wakefulness & $\begin{array}{l}\text { Increases from NREM, depending } \\
\text { on brain region }\end{array}$ \\
\hline Respiration & Decreases from wakefulness & $\begin{array}{l}\text { Increases and varies from NREM, } \\
\text { but may show brief stoppages; } \\
\text { coughing suppressed }\end{array}$ \\
\hline Airway resistance & Increases from wakefulness & $\begin{array}{l}\text { Increases and varies from } \\
\text { wakefulness }\end{array}$ \\
\hline Body temperature & $\begin{array}{l}\text { Is regulated at lower set point } \\
\text { than wakefulness; shivering } \\
\text { initiated at lower temperature } \\
\text { than during wakefulness }\end{array}$ & $\begin{array}{l}\text { Is not regulated; no shivering or } \\
\text { sweating; temperature drifts } \\
\text { toward that of the local } \\
\text { environment }\end{array}$ \\
\hline Sexual arousal & Occurs infrequently & Greater than NREM \\
\hline
\end{tabular}

Figure 4: Sleepphisiology.

Part III. Source: https://www.slideshare.net/mabdelghani/physiology-ofsleep-and-eeg-for-undergraduates 


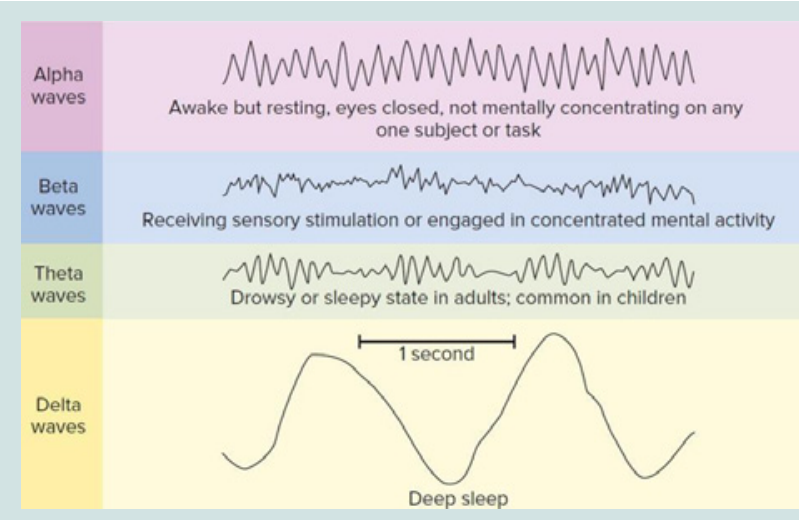

Figure 5: Sleepwaves.

Source:https://corrosion-doctors.org/Dreaming\%20is\%20Personal/Glossary. $\mathrm{htm}$

on the same day. These episodes must have occurred at least three times a week in the last 3 months. In order for there to be a diagnosis of narcolepsy, episodes of cataplexy must occur at least a few times a month, characterized by a sudden, brief and reversible episode of muscle weakness that occurs in conjunction with emotional stimuli, such as laughter, surprise, anger, joy or sadness.

\section{Respiratory-related sleep disorders}

a) Obstructive sleep apnea / hypopnea: repeated episodes of obstruction of the upper airway (pharyngeal) during sleep that can manifest itself with nocturnal respiratory disorders or daytime sleepiness, asthenia or non-restorative sleep despite sufficient opportunity to sleep and not explained by other mental disorder or medical condition. Furthermore it is necessary that there are polysomnographic evidences of 15 or more apnea and/or obstructive hypopnea as per hour of sleep.

b) Central sleep apnea: repeated episodes of apnea and hypopnea during sleep caused by a change in respiratory effort. These are ventilation control disorders in which respiratory events occur with a periodic or intermittent pattern. This disorder is detectable when polysomnographic evidences of 5 or more central apnea are present per hour of sleep and when the disorder is not better explained by another concomitant sleep disorder.

c) Sleep-relatedhypoventilation: polysomnography shows episodes of decreased respiration associated with high levels of carbon dioxide, in the absence of a concomitant sleep disorder.

Circadian disorders of the wake-sleep rhythm: sleep interruption due to an alteration of the circadian system, to a misalignment of the endogenous circadian rhythm and the wake-sleep rhythm required by the physical conditions of an individual or imposed by social or work commitments. In these cases, sleep interruption leads to excessive sleepiness or insomnia or both. Sleep disturbance causes clinically significant distress or impairment in social, occupational or other important areas.

Parasomnias: they are disorders characterized by abnormal experiences and behaviors or physiological events that occur in association with sleep, specific stages of sleep or sleep-wake passages. According to the most recent classification of sleep disorders, parasomnias represent a large and heterogeneous group of sleep disorders that consist of "undesirable manifestations that accompany sleep and that often seem aimed at achieving a goal. In some cases they can cause trauma and disturb sleep (of the patient or of those around him)". The different forms of parasomnia are classified according to their occurrence during the different phases of sleep:

- NREM sleeps parasomnias (arousal disorders). NREM sleep, especially during the deep sleep for this reason, these manifestations occurs more frequently within 1-2 hours of falling asleep. An episode on average last few minutes but its duration can be very variable: from a few seconds up to even 30 minutes. Usually, NREM sleep parasomnias arise in childhood (probably two to the high representation of deep sleep during this phase of life) and tend to shrink or disappear with adulthood. There is often familiarity with such episodes, which can be triggered by certain factors such as sleep deprivation, irregular sleep-wake cycles, fever, infections, alcohol, certain medications and other sleep disorders including sleep apnea. Patient soften do not retain any memory of the episodes themselves, the clinical features of which can be very heterogeneous. We distinguish 3 different types of manifestations (which can also occur in the same subject) which, according to the most recent interpretations, represent a continuum of the same phenomenon, with different degrees of complexity:

a) Confusion awake up. Episodes of partial wakening not associated with walking or autonomic disorders (the child seems to be awake but confused, disoriented, sometimes aggressive, does not respond adequately to orders, can speak but not in an inconsistent way);

b) Sleepwalking. Episodes characterized by more or less complex automatic behavior (like walking, eating, drinking, and leaving home ...);

c) Night terrors. Episodes of partial wakening, often with sudden onset, with expression of terror, intense agitation, sweating, pallor, wheezing, tachycardia.

\section{- Parasomnias usually associated with REM sleep}

They are complex motor manifestations that occur during REM sleep:

\section{a) Behavioral disorder in REM}

REM sleep represents that phase of sleep, mostly represented in the second part of the night, in which there is an almost complete loss of tone of the voluntary musculature ("it is as if immobilized") and during which the more dreamlike activity occurs intense. The behavioral disturbance in REM sleep (RBD) is characterized by the loss of physiological muscle a tony. For this reason, during the episodes, which occur more frequently in the second part of the night, the patients present an excessive motor activity, often characterized by abrupt behavior (such as screaming, punching and kicking), in relation to the content of their dreams. In fact, patients often report dreams with a negative content, which they "act" by performing violent actions, which can assume characteristics of aggressiveness, for example towards the bed partner. These manifestations therefore entail a high risk of trauma both for the patient and for those close to him. Their duration is usually between 2 and 10 minutes and the frequency can be very varied: from weekly or monthly episodes to 
multi-night (4-5 / night).

\section{b) Sleep paralysis}

They consist in the inability to perform any voluntary motor activity (one has the perception of being completely immobilized), although the subject is completely conscious. They can occur during the phase of falling asleep ("hypnagogic paralysis") or following an awakening ("hypnopompic paralysis"). They can be accompanied by auditory or visual hallucinations and can last from a few seconds to several minutes, often causing intense anxiety in the person who lives them. They can be resolved spontaneously or following sensory stimulation. They can be favored by an irregular sleep-wake rhythm and sleep deprivation.

\section{c) Nightly nightly}

They consist of fearful dreams, with a negative content, often of long duration; these dreams frequently induce the awakening of the subject that keeps a vivid memory of it. They are common in children or in patients with "post-traumatic stress disorder". They can be favored by fever or by the abrupt withdrawal of alcohol or drugs that reduce REM sleep (amphetamines, some antidepressants and benzodiazepines). These conditions, in fact, could lead to a sharp and significant increase in the representation of REM sleep, favoring the occurrence of nightmares.

- Other parasomnias.

\section{a) Groaning (Catathrenia)}

It consists of the emission of a monotonous vocalization during a prolonged exhalation associated with bradypnea (reduction of respiratory rate). It often arises at a young age (20-30 years). The episodes, which last about 2-20 seconds, occur more frequently during REM sleep (especially in the second half of the night). The cause still appears to be unknown and, at present, there is no treatment. Cases have been described in association with sleep apnea and only a few have been resolved with nocturnal ventilatory treatment. However, it is important to distinguish such manifestations from snoring episodes.

\section{b) Exploding head syndrome}

It is characterized by the perception of a sound often very intense, similar to an explosion or an explosion, which occurs especially during the phase of falling asleep, often resulting in a sudden wakening. The perceived sound, although very violent, is never accompanied by pain, but sometimes it can be related to visual flashes. If the crises are recurrent and the sound perceived is particularly intense, the patient will tend to fall asleep with fatigue for fear of a new attack. The syndrome affects mainly women around the age of 50 and can be favored by stressful conditions. Currently there is no univocal explanation of these phenomena as well as an effective therapy (some cases described have been treated with calcium channel blockers).

\section{c) Hypnagogical / Ipnopompical hallucinations.}

Vivid experiences, similar to dreams, often with bizarre or terrifying contents, which occur during sleep ("hypnagogic") or after awakening ("hypnopompic"). During the attacks the fantastic sensations can be mistaken for real. In most cases these are visual hallucinations, but they can also be auditory, tactile, gustatory or olfactory. They may be associated with sleep paralysis and, like these, occur frequently in individuals without other sleep disorders or may be one of the symptoms of narcolepsy.

\section{d) Sleep-related eating disorder (SRED).}

Consists of repeated episodes during sleep of compulsive food or drink ingestion (even unusual or inedible); often the level of consciousness during the episodes appears to be null or partial, just as the patient on waking frequently does not retain any memory of what happened. This form of parasomnias appears more frequent in the female sex and has an onset around the 20-30 years; familiarity with other NREM sleep parasomnias is common. SRED can be associated with other sleep disorders (arousal disorders, restless legs syndrome, sleep apnea, narcolepsy) and can be triggered by taking certain drugs (benzodiazepines, zolpidem, lithium) or by abrupt withdrawal of alcohol intake. This syndrome must be distinguished from other forms of eating behavior in sleep such as "Night Eating Syndrome", characterized by hyperphagia (overeating) evening and night and / or anorexia (lack of appetite) morning and insomnia.

\section{The Neural Correlates in Sleep-Wake Disorders}

A first system that controls and maintains the waking state is represented by the aminergic nuclei of the brainstem [7], in particular by the noradrenergic neurons of the locus coeruleus and by the serotonergic neurons of the raphe nuclei, but it is assumed that the substance dopaminergic neurons also play a role Black. These neurons project diffusely to the cortex, the thalamus, the hypothalamus and the hippocampus. When the subject is alert, the discharge frequency of the neurons of these systems is maximum, is greatly reduced during non-REM sleep and almost completely during REM sleep, suggesting that they are systems involved in waking maintenance. These neurons can also undergo phenomena of self-inhibition that promote sleep. Conditions that stimulate activity promote wakefulness, but if these systems are inhibited, sleep is promoted. If, however, it seems true that the stimulation of the noradrenergic system stimulates and maintains wakefulness, serotonin, while also stimulating wakefulness, favors, over time, the synthesis and release of substances that promote sleep and inhibit the cholinergic neurons of the forebrain basal, involved in maintaining the vigil, thus playing an ambiguous role.

A second system that promotes wakefulness is the cholinergic neurons of the basal forebrain. These neurons project to the cortex, activating it, to the hippocampus and amygdale, and, in addition to being awake; they are active during the REM phase, which are not very active in the non-REM phase. They are inhibited by serotoninergic terminations originating from the raphe nuclei. The cholinergic nuclei of the brainstem include the laterodorsal nucleus of the pontine tegmentum and the peduncolo pontine tegmentum nucleus which are made up of two populations of neurons. A first population is characterized by neurons active during REM sleep, which discharge watery low frequency during wakefulness and non-REM sleep and which project to the aminergic nuclei of the brainstem. The second population consists of neurons whose discharge frequency is maximum during wakefulness and during REM sleep and which project to the thalamus and hypothalamus, activating them. The tuberomammillary nucleus contains Histaminergic hypothalamic 
neurons which project diffusely to almost the entire central nervous system, promoting waking maintenance and are maximally active in this phase. The inhibition of these neurons with antihistamine induces sleepiness. The posterolateral hypothalamus comprises a small group of orexinergic neurons that maintain vigil and are also involved in the regulation of food intake. They diffusely project to the structures involved in the regulation of the sleep-wake cycle in the central nervous system.

Vigil is a behavioral state characterized by arousal and cortical activation, manifested in a desynchronized EEG pattern. In humans it is characterized by a beta-type rhythm (frequency: $15-30 \mathrm{~Hz}$; amplitude: $<20 \mu \mathrm{V}$ ) during active and alpha-type vigil (frequency: 8 $12 \mathrm{~Hz}$; amplitude: $<50 \mu \mathrm{V}$ ) during a relaxed vigil (Figure 5).

This behavioral state is supported by the interaction between different brain regions and by different types of neuromedicators:

1) Glutamate (Glu). At the level of the brainstem, the Reticular Formation (FR) is essential to maintain the typical activating characteristics of waking. The FR is part of the Ascending Activating System (ARAS) which through diffuse projections from the brainstem reaches up to the cortex, causing desynchronization. The projections that depart from the neurons present in the oral part of the pontine and mesencephalic FR ascend towards the prosencephaly and the cortex, where they sustain a cortical bone through a dorsal pathway, towards the thalamus, and a ventral pathway, towards the hypothalamus and the fore brain basal (Lindsley et al., 1950; Starzl et al., 1951). On the other hand, the neurons present in the caudal part of the pontine and bulbar FR facilitate the tone of the postural muscles through their projections towards the motor neurons present in the spinalcord (Jones, 2005). One of the neuromediators that is involved in projections from FR nuclei is probably Glutamate (Glu) (Jones, 1995). The importance of glutamate in wakefulness is also underlined by the fact that most anesthetics (including those for inhalation and ketamine) attenuate glutamate-mediated neuro transmissions (Rudolph and Antkowiak, 2004).

2) Nore Pinephrine (NA). The Locus Coeruleus (LC) is another important structure for waking: its noradrenergic neurons stimulate cortical activation and arousal through their diffuse projections to the forebrain, the trunk of the brain and the spinalcord. The neurons of the LC, in fact, discharge every frequently during wakefulness (especially during active wakefulness), decrease their discharge during slow-wave sleep and cease their activity during REM sleep (Aston-Jones and Bloom, 1981; McCarley and Hobson, 1975). The role of Nor Adrenaline (NA) is however ambivalent, as it depends on the type of receptor on which it is going to act; in general, $\alpha-1$ adrenergic receptors have an excitatory action (depolarization due to the closure of potassium channels); on $\alpha-2$ adrenergic receptors, it has an inhibitory action (hyper polarization due to opening of the potassium channels). Through its receptors, the NA selectively excites the other systems that are involved in waking and inhibits those structures that support sleep, especially at the level of the basal prosencephalon and the preoptic area (Jones, 2005).

3) Dopamine (DA). At the most rostral level, in the mesencephalic region, the Substantia Nigra (SN), the ventral tegmental area (VTA) and the ventral Periaqueductal Gray (vPAG) play a priority role in attentional processes and in maintaining arousal, through direct dopaminergic connections towards the striatum nucleus, the basal forebrain and the cortex ( $\mathrm{Lu}$ et al., 2006). These dopaminergic neurons show the highest level of activity during wakefulness and REM sleep, 33 while their discharge decreases during slow-wave sleep (Lena et al., 2005; Maloney et al., 2002).

4) Serotonin (5-HT). The waking state is also maintained by the neurons of other brain stem structures: the Dorsal Nucleus of the Raphe (DR) and the Medial Nucleus of the Raphe (MR). The neurons of these structures use serotonin (5-HT) as a neurotransmitter and project towards many regions of the diencephalon, the limbic system and the neocortex. These structures reach their maximum peak discharge during wakefulness, decrease their activation during NREM sleep and are silent during REM sleep.

5) Acetylcholine (Ach). Ponto-mesencephalic cholinergic structures, such as the nucleus of the Laterodorsal tegmentum (LDT) and the nucleus of the Pedunculopontine Tegmentum (PPT), play an important role in maintaining wakefulness, but also in REM sleep, facilitating arousal cortical and desynchronization. Both structures, parallel to the neurons of the FR, project towards the specific thalamus-cortical projection system, where they cause a diffuse cortical activation (Jones, 1995; McCormick, 1992; Steriade et al., 1990). In small part, these structures also project to the posterior hypothalamus, to the basal pros encephalon and to the FR through the extra-thalamic pathway. The discharge activity of these cholinergic neurons of LDT and PPT is high during wakefulness, decreases during NREM sleep and increases again during REM sleep (elMansari M. et al., 1989; Steriade et al., 1990). The discharge of this cholinergic system occurs in association with states of cortical activation (Jones, 2005; Steriade et al., 1990), but it is not related to behavioral arousal. In fact, it has been shown that the injection at the level of the pontomesencephalic tegmentum of Acetyl Choline Agonists (ACh), such as carbachol, causes cortical activation accompanied by inhibition of muscle tone (Jones, 2004b). According to this, ACh can act in different populations of target cells to promote cortical activation and inhibit muscle tone. In the thalamus, for example, ACh acts on nicotinic receptors (nAChRs) and muscarinic receptors (M1ACh and M2ACh) to facilitate cortical activation (Curro et al., 1991; McCormick, 1992); in fact, Ach activates the thalamus-cortical relais nuclei through an excitatory action directed on the nAChRs and $\mathrm{M} 1 \mathrm{ACh}$ receptors and through an indirect facilitation: inhibitory action on the thalamic-reticular GABAergic neurons through the M2ACh receptors. Similarly, in the FR of the brain stem, ACh can act on different receptors to excite some neurons that are involved it her in cortical activation or motor inhibition, and based on two behavioral states, the wake and REM sleep, which are similar to each other due to the desynchronization of the tracing, but differ in other aspects, such as the presence or absence of muscular tony. How can we explain this phenomenon? Under normal conditions there is a certain balance between the noradrenergic and cholinergic systems so that the activation of both neuronal systems maintains the waking state, characterized by activation of the motor system and cortical arousal. REM sleep and loss of muscle tone occur when the noradrenergic system is inactive, while cholinergic is active. This association became evident in the 1970s in an animal model through the administration of Acetyl Cholinesterase Inhibitors (AChE), used to strengthen the 
activity of Ach. When they were administered alone, AchE inhibitors stimulated wakefulness; conversely, when they were administered following the removal of catecholamines (therefore also of NA), by administration of reserpine, they stimulated REM sleep (Curro et al., 1991; Jones, 2004b; McCormick, 1992). In men, acetyl cholinesterase inhibitors, when given during waking, stimulate cortical activation and induce prolongation of waking state; while, they accelerate the onset of REM sleep when administered during NREM sleep, when the noradrenergic system and other arousal systems are inactive (Gillin and Sitaram, 1984). On a more rostra level, in the basal pros encephalon, there are other neural compartments that use acetylcholine as a neuro mediator, such as the medial septum (MS / vDB: medial septum / vertical limb of the diagonal band) and other nuclei, which play a major role in cortical activation, receiving input from structures of the brainstem and the hypothalamus, and projecting diffusely towards the cortex (Jones, 2004a; Lee et al., 2005). These neurons are very important in generating the theta rhythm (4 $7 \mathrm{~Hz})$ and gamma $(30-60 \mathrm{~Hz})$ during wakefulness and REM sleep. At this level there are also other GABAergic and glutamatergic neurons that increase their discharge in association with corticalactivation (Gritti et al., 1997; Manns et al., 2003) and seem, above all the latter, to be responsible for the increase in behavioral arousal and tone of postural muscles (Gritti et al., 1994; Henny and Jones, 2006).

6) Histamine (His). As already observed by von Economo (von Economo, 1930), another important region for waking maintenance is the hypothalamus. The back and side of this structure appears to have a specific role in cortical activation. The neurons of the tuberomammillary nuclei (TMN), forming part of the posterior rhypothalamus, use histamine as mediator and stimulate, through diffuse projections, cortical activation (Brown et al., 2001b; Saper et al., 2001). Histaminergic neurons discharge profusely during wakefulness, decrease during NREM sleep and cease their activity in REM sleep. Histamine has an excitatory effect on most of the ARAS nuclei and, in contrast, inhibits the "sleep active" neurons of the Ventro Lateral Preoptic area (VLPO), by excitatory synapses on inhibitory inter neurons (Liu et al., 2010).

7) Oressin / Hypocretin (Orx / Hcrt).Finally, at the level of the lateral hypothalamus (Lateral Hypothalamus, LH) there are neurons that use a known peptide with the double word: oressin / hypocretinas mediator. This plays a fundamental role in promoting and stabilizing the waking state, while suppressing REM sleep. A deficiency of this peptide leads to narcolepsy (Chemelli et al., 1999; Lin et al., 1999; Mignot et al., 2002; Peyron et al., 2000), ie a neurological disorder characterized by daytime sleepiness accompanied by cataplexy (sudden loss of muscle tone), hypnagogic hallucinations (auditory and / or visual hallucinations during the phases of sleep / wake or sleep) and sleep paralysis (inability to move or speak during awakenings) (Yoss and Daly, 1957). Oressinergic neurons have connections with all the nodes that intervene in the wake-sleep cycle and are actively inhibited during the NREM sleep phase by the GABAergic neurons of the preoptic region and of the basal pros encephalon. These oressinergic nuclei maintain the vigil by a diffuse projection towards the aminergic systems, in particular towards the LC, where they promote the vigilat the expense of REM sleep (Bourgin et al., 2000; Hagan et al., 1999). The anatomical distribution of the connections of oressinergic neurons and their involvement in narcolepsy has led to the hypothesis that orexin, besides being important for waking maintenance, is fundamental for the stabilization of the wake-sleep switch (Saper et al., 2001).

\section{Melatonin: Contexts and Clinical Profiles}

From a neurobiochemical point of view, as already stated [8], the pineal gland produces melatonin, a hormone isolated for the first time in 1958 by Aaron Lerner and produced by pinealocytes starting from the neurotransmitter serotonin (5-hydroxy-tryptamine) for $\mathrm{N}$-acetylation and oxy-methylation, by virtue of the fact that these cells contain the enzyme Hydroxyindole-O'xymethyltransferase (HIOMT), epiphysis marker enzyme.

It acts in the circadian rhythm of sleep and has powerful antioxidant effects: melatonin is synthesized in the absence of light from the pineal gland; shortly after the onset of darkness, its concentrations in the blood increase rapidly and reach the maximum between 2 and 4 am and then gradually decrease as the morning approaches.

Exposure to light (especially at the blue wave length between 460 and $480 \mathrm{~nm}$ ) inhibits the production of melatonin in a dosedependent manner. It is therefore used for the short-term treatment of insomnia over 55 years of age.

The side effects of melatonin are not null, although the contrary belief is widespread: over the years, various professional bodybuilders and various sports information magazines have affirmed the possibility, with the support of some scientific studies, that daily doses between $0.5 \mathrm{mg}$ and $3 \mathrm{mg}$, taken 30-60 minutes before training, increase the levels of growth hormone, without giving side effects, which are usually recognized in irritability and drowsiness.

Melatonin decreases the release of GnRH: for this reason the synthesis of testosterone and therefore libido decreases. More precisely, it inhibits the secretion of the luteinizing hormone, which stimulates the male endocrine activity of the interstitial cells of the testis with testosterone and sperm production, and in the female ovulation and conversion of the ovarian follicle into the corpus luteum. Taken for prolonged periods, melatonin can have a depressive effect in predisposed subjects; furthermore, it can inhibit ovulation precisely because of the suppression of the GnRH release it causes.

Recent research in the biomedical field, especially on melatonin, has shown that:

1) Melatonin is importantly involved in inflammatory processes and cellular apoptosis [9].

2) Exposure to electromagnetic fields decreases the secretion of melatonin [10], which negatively affects cellular processes linked to death, acts on sex hormones and connected glands and interferes with the sleep-wake rhythm [11].

3) Melatonin intervenes in the neurobiological processes involved in anorexic and bulimic disorders and in the predisposition to be subject to these psychophysical pathologies [12].

4) Melatonin is involved with cortisol in the immunomodulatory response [13]. 
5) Melanin intervenes in the regulation of acidosis in malignant tumor processes [14]

6) Melatonin is a potent inhibitor of ovarian and prostate cancer $[15,16]$.

7) Melatonin has positive effects on blood pressure, reducing hypertension [17].

8) Melatonin, having antioxidant and modulating properties of the circadian rhythm, has positive effects on drug therapy in the presence of schizophrenia and in general in psychotic syndromes $[18,19]$.

9) Melatonin has a positive effect on blood sugar, reducing blood levels and favoring a positive prognosis on insulinic therapy in the rats [20].

10) Melatonin, compared to problems related to the central nervous system, seems to be directly involved in the reduction of tissue and nerve lesions, affecting free radicals due to its powerful antioxidant effect. Since endogenous melatonin levels decrease significantly in senility, these result simply that the loss of this antioxidant could contribute to the incidence or severity of some agerelated neurodegenerative diseases [21].

11) Melatonin, precisely because it is related to serum and ionized calcium levels [22], positively intervenes in vertebral disorders and intervertebral degeneration (in chicken) $[23,24]$; also in the cartilaginous problems and curvature of scoliosis $[25,26]$.

12) Melatonin has beneficial effects on respiratory problems linked to obstructive bronchospasm [27].

In recent decades $[28,29]$, the integration of melatonin in the diet has allowed a significant and positive management of primary sleep disorders, especially at a dose of 2-10 mg, unlike the classic $0,5-1 \mathrm{mg}$ dose indicated for jet lag disorder.

\section{Conclusion}

Sleep disorders represent a primitive alteration of its regulatory mechanisms and therefore of the physiological rhythms of the sleepwake cycle or be the clinical expression of other pathologies, organic or psychic. Chronic sleep deprivation, altering the sleep-wake cycle e all the associated biological rhythms, involves the exhaustion of energy that feeds our life, compromising its quality in all its dimensions: personal, affective, family, socio-relational and working. Sleep is therefore a cornerstone of health.

For this reason, morphological disorders must always be taken seriously consideration and shared with your doctor to be able to identify and therefore eliminate, where possible, any causes triggers, through a targeted behavioral and / or intervention pharmacological. The so-called "sleep hygiene", which is achieved through the adoption of behaviors that aim to promote a good night's sleep, already helps improve sleep quality and maintain it over time, representing the primary strategy.

The basic advice, in the absence of diseases, to sleep properly are:

- Respect your own sleep-wake rhythm, lying down and waking up around the same time (even during the weekend) and avoiding naps during the day.

- Always follow the same ritual before going to bed, dedicating yourself to relaxing activities.

- Perform physical activity during the day and not in the evening hours.

- Take meals at regular times, preferring a light diet for dinner.

- Avoid the intake of exciting substances (tea, coffee, alcohol, nicotine) in the evening hours.

- Rest on a comfortable bed in a cool, dark room silent and well ventilated.

In the presence of a pathological disorder, however, the intervention of a specialist in neurology or psychiatry is required to carry out a targeted therapy consisting of pharmacological and/or psychotherapeutic treatment.

\section{References}

1. Giulio Perrotta (2019) Psicologia clinica. Lux-co Edn. Clin Psychol A5: 244.

2. Giorgio Coccagna, II sonno e i suoi disturbi, Piccin-Nuova Libraria ( $2^{\text {nd }}$ edn).

3. Sónno, in Treccani.it - Vocabolario Treccani on line, Istituto dell'Enciclopedia Italiana, 2011

4. Ltd. Pan Macmillan. Macmillan dictionary for student's pp. 1216.

5. Hobson JA, Pace-Schott EF, Stickgold R (2000) Dreaming and the brain: Toward a cognitive neuroscience of conscious states. Behav Brain Sci 23: 793-842.

6. Giulio Perrotta (2019) Psicologia clinica. Lux-co Edn. Clin Psychol A5: 244.

7. Imeri L, Mazzanti M (2013) Sonno e malattie neurodegenerative in modelli animali. Tesi di Dottorato di Ricerca 1-239.

8. Perrotta G (2019) The Pineal Gland: anatomical, clinical and neurobiochemical profiles, between hypotheses of the past, certainties of the present and future perspectives. J Neurol Psychol 7: 1-5.

9. Tarocco A, Caroccia N, Morciano G, Wieckowski MR, Ancora G, et al. (2019) Melatonin as a master regulator of cell death and inflammation: molecular mechanisms and clinical implications for newborn care. Cell Death Dis 10: 317.

10. Pfluger DH, Minder CE (1996) Effects of exposure to $16.7 \mathrm{~Hz}$ magnetic fields on urinary 6-hydroxymelatonin sulfate excretion of Swiss railway workers. J Pineal Res 21: 91-100.

11. Halgamuge MN (2019) Pineal melatonin level disruption in humans due to electromagnetic fields and ICNIRP limits. Radiat Prot Dosimetry 154: 405416.

12. Liu L, Yi J, Ray WK, Vu LT, Helm RF, et al. (2019) Fasting differentially alters the hypothalamic proteome of chickens from lines with the propensity to be anorexic or obese. Nutr Diabetes 9:13.

13. Baekelandt S, Mandiki SNM, Kestemont P (2019) Cortisol and melatonin involved in the immune modulation by the light. J Pineal Res 29: e12573.

14. Sonehara NM, Lacerda JZ, Jardim-Perassi BV, de Paula Jr R, MoschettaPinheiro MG, et al. (2019) Melatonin regulates tumor aggressiveness under acidosis condition in breast cancer cell lines. Oncol Lett 17: 1635-1645.

15. Zare H, Shafabakhsh R, Reiter RJ, Asemi Z (2019) Melatonin is a potential inhibitor of ovarian cancer: molecular aspects. J Ovarian Res 12: 26.

16. Hogan E, Almira-Suarez I, Li S, Collins SP, Jean WC (2019) Clinical management of prostate cancer metastasis to pineal gland: case report and review of literature. World Neuro Surg 122: 464-468.

17. Hadi A, Ghaedi E, Moradi S, Pourmasoumi M, Ghavami A, et al. (2019) 
Effects of melatonin supplementation on blood pressure: a systematic review and meta-analysis of randomized controlled trials. Horm Metab Res 51: 157164.

18. Bastos MAV Jr, Oliveira Bastos PRH, Portella RB, Soares LFG, Conde RB et al. (2019) Pineal gland and schizophrenia: A systematic review and metaanalysis. Psychoneuroendocrinology 104: 100-114

19. Takahashi T, Nakamura M, Sasabayashi D, Nishikawa $Y$, Takayanagi $Y$ (2018) Reduced pineal gland volume across the stages of schizophrenia. Schizophr Res 2016: 163-170.

20. Dantas-Ferreira RF, Raingard H, Dumont S, Schuster-Klein C, GuardiolaLemaitre B, et al. (2018) Melatonin potentiates the effects of metformin on glucose metabolism and food intake in high-fat-fed rats. Endocrinol Diabetes Metab 1: e00039.

21. Uyanikgil Y, Cavusoglu T, DoganKılıc K, Yigitturk G, Celik S (2017) Usefu effects of melatonin in peripheral nerve injury and development of the nervous system. J Brachial Plex Peripher Nerve Inj 12: e1-e6.

22. Chen Y, Wu Y, Shi H, Wang J, Zheng Z, et al. (2019) Melatonin ameliorates intervertebral disc degeneration via the potential mechanisms of mitophagy induction and apoptosis inhibition. J Cell Mol Med 23: 2136-2148.

23. Alini M, Eisenstein SM, Ito K, Little C, Kettler AA, et al. (2008) Are animal models useful for studying human disc disorders/degeneration? Eur Spine J 17: 2-19.Turgut M, Başaloğlu HK, Yenisey C, Özsunar Y (2006) Surgical pineal ectomy accelerates intervertebral disc degeneration process in chicken. Eur Spine J 15: 605-612.

24. Uyanikgil Y, Cetin Uyanikgil EO, Turgut M (2016) Effects of Melatonin upon vascularity of cartilage end plate of intervertebral Disc. J Spine 5:e123.

25. Liu H, Liu Z, Chi-wai M, Guo J, Han X, et al. (2019) The effect of exogenous melatonin on reducing coliotic curvature and improving bone quality in melatonin-deficient, C57BL/6J mice. Sci Rep 9: 6202.

26. Tsai SC (2017) Chronic obstructive pulmonary disease and sleep related disorders. Curr Opin Pulm Med 23: 124-128.

27. Claustrat B, Leston J (2015) Melatonin: Physiological effects in humans. Neurochirurgie 61: 77-84.

28. Quera-Salva MA, Claustrat B (2018) Melatonin: physiological and pharmacological aspects related to sleep: The interest of a prolonged-release formulation (Circadin ${ }^{\circledR}$ ) in insomnia. Encephale 44: 548-557. 